Novi Sad J. Math.

Vol. 42, No. 1, 2012, 49-57

\title{
COMMON FIXED POINT THEOREM IN QUASI-UNIFORMIZABLE SPACES
}

\section{Tatjana Došenović ${ }^{\boxplus}$}

\begin{abstract}
In this paper a common fixed point theorem in sequentially complete Hausdorff quasi-uniformizable spaces is proved. Using the additional condition for the quasi-metric $d_{\lambda}$ the condition (2) of Theorem 3.92 in the paper [4] is improved. Since Menger space $(S, \mathcal{F}, T)$, where $\sup T(a, a)=1$ is a quasi-uniformizable space a corollaries on common $a<1$ fixed points in Menger spaces is obtained.
\end{abstract}

AMS Mathematics Subject Classification (2010): 54H25, 55M20, 47H10

Key words and phrases: t-norm, quasi-uniformizable space, common fixed point, Menger space

\section{Introduction}

D.H. Tan in [8] introduced the definition of quasi-uniformizable spaces. Using a similar method as in [4] (see also [2], [3] and [y]) we prove in this paper a common fixed point theorem for three mappings in sequentially complete Hausdorff quasi-uniformizable spaces. As a corollary we prove a common fixed point theorem in Menger spaces. Menger spaces (see [6]) are a generalization of the notion of a metric space $(M, d)$ in which the distance $d(p, q)(p, q \in M)$ between $p$ and $q$ is replaced by a distribution function $F_{p, q} \in \Delta^{+} . F_{p, q}(x)$ can be interpreted as the probability that the distance between $p$ and $q$ is less than $x$. Since then, the theory of probabilistic metric spaces has been developed in many directions $([\mathbb{[}],[[]])$.

\section{Preliminaries}

D.H. Tan in $[8]$ introduced the notion of quasi-uniformizable spaces.

Definition 1. Let $S$ and $I$ be arbitrary sets, $g: I \rightarrow I$ and for every $i \in I$, $d_{i}: S \times S \rightarrow \mathbb{R}^{+}$. The triplet $\left(S,\left(d_{i}\right)_{i \in I}, g\right)$ is said to be a quasi-uniformizable space if for every $x, y, z \in S$ and $i \in I$ the following hold:

a) $d_{i}(x, y) \geq 0, d_{i}(x, x)=0$

b) $d_{i}(x, y)=d_{i}(y, x)$

c) $d_{i}(x, y) \leq d_{g(i)}(x, z)+d_{g(i)}(z, y)$.

\footnotetext{
${ }^{1}$ Faculty of Technology, University of Novi Sad, Bulevar Cara Lazara 1, Novi Sad, Serbia, e-mail: tatjanad@tf.uns.ac.rs
} 
A quasi-uniformizable space $\left(S,\left(d_{i}\right)_{i \in I}, g\right)$ is Hausdorff if the relation $d_{i}(x, y)=0$, for all $i \in I$ implies that $x=y$. A Hausdorff quasi-uniformizable space $\left(S,\left(d_{i}\right)_{i \in I}, g\right)$ becomes a Hausdorff topological space if the fundamental system of neighbourhoods of $x \in S$ is given by the family $\mathcal{U}_{x}=(B(x, \varepsilon, i))_{i \in I}^{\varepsilon>0}$

$$
B(x, \varepsilon, i)=\left\{y \mid y \in S, d_{i}(x, y)<\varepsilon\right\}, \quad i \in I, \varepsilon>0 .
$$

Let $\Delta^{+}$be the set of all distribution functions $F$ such that $F(0)=0(F$ is a nondecreasing, left continuous mapping from $\mathbb{R}$ into $[0,1]$ such that $\sup _{x \in \mathbb{R}} F(x)=$ $1)$.

The ordered pair $(S, \mathcal{F})$ is said to be a probabilistic metric space if $S$ is a nonempty set and $\mathcal{F}: S \times S \rightarrow \Delta^{+} \quad\left(\mathcal{F}(p, q)\right.$ written by $F_{p, q}$ for every $(p, q) \in S \times S)$ satisfies the following conditions:

1. $F_{u, v}(x)=1$ for every $x>0 \Rightarrow u=v(u, v \in S)$.

2. $F_{u, v}=F_{v, u}$ for every $u, v \in S$.

3. $F_{u, v}(x)=1$ and $F_{v, w}(y)=1 \Rightarrow F_{u, w}(x+y)=1$ for $u, v, w \in S$ and $x, y \in \mathbb{R}^{+}$.

A Menger space (see []]) is an ordered triple $(S, \mathcal{F}, T)$, where $(S, \mathcal{F})$ is a probabilistic metric space, $T$ is a triangular norm (abbreviated t-norm), and the following inequality holds

$F_{u, v}(x+y) \geq T\left(F_{u, w}(x), F_{w, v}(y)\right)$ for every $u, v, w \in S$ and every $x>0, y>0$.

Recall that a mapping $T:[0,1] \times[0,1] \rightarrow[0,1]$ is called a triangular norm (a t-norm) if the following conditions are satisfied:

(i) $T(a, 1)=a$ for every $a \in[0,1]$,

(ii) $T(a, b)=T(b, a)$ for every $a, b \in[0,1]$,

(iii) $a \geq b, c \geq d \Rightarrow T(a, c) \geq T(b, d) \quad(a, b, c, d \in[0,1])$,

(iv) $T(a, T(b, c))=T(T(a, b), c) \quad(a, b, c \in[0,1])$.

The following are the four basic t-norms:

$$
\begin{aligned}
& T_{M}(x, y)=\min (x, y) \\
& T_{P}(x, y)=x \cdot y \\
& T_{L}(x, y)=\max (x+y-1,0) \\
& T_{D}(x, y)= \begin{cases}\min (x, y) & \text { if } \max (x, y)=1, \\
0 & \text { otherwise. }\end{cases}
\end{aligned}
$$

As regards the pointwise ordering, we have the inequalities

$$
T_{D}<T_{L}<T_{P}<T_{M} .
$$

The $(\epsilon, \lambda)$-topology in $S$ is introduced by the family of neighbourhoods $\mathcal{U}=\left\{U_{v}(\epsilon, \lambda)\right\}_{(v, \epsilon, \lambda) \in S \times \mathbb{R}_{+} \times(0,1)}$, where

$$
U_{v}(\epsilon, \lambda)=\left\{u ; F_{u, v}(\epsilon)>1-\lambda\right\} .
$$


If a t-norm $T$ is such that $\sup _{x<1} T(x, x)=1$, then $S$ is in the $(\epsilon, \lambda)$-topology a metrizable topological space.

In [T], the following class of t-norms is introduced, which is useful in the fixed point theory in probabilistic metric spaces.

Let $T$ be a t-norm and $T_{n}:[0,1] \rightarrow[0,1](n \in \mathbb{N})$ is defined in the following way:

$$
T_{1}(x)=T(x, x), T_{n+1}(x)=T\left(T_{n}(x), x\right)(n \in \mathbb{N}, x \in[0,1]) .
$$

We say that t-norm $T$ is of the $H$-type if $T$ is continuous and the family $\left\{T_{n}(x)\right\}_{n \in \mathbb{N}}$ is equicontinuous at $x=1$.

The family $\left\{T_{n}(x)\right\}_{n \in \mathbf{N}}$ is equicontinuous at $x=1$, if for every $\lambda \in(0,1)$ there exists $\delta(\lambda) \in(0,1)$ such that the following implication holds:

$$
x>1-\delta(\lambda) \Rightarrow T_{n}(x)>1-\lambda, \text { for all } n \in \mathbb{N} .
$$

A trivial example of t-norms of $H$-type is $T=T_{M}$. A nontrivial example is given in the paper [T].

Each t-norm $T$ can be extended (by associativity) (see [5]) in a unique way to an $n$-ary operation taking for $\left(x_{1}, \ldots, x_{n}\right) \in[0,1]^{n}$ the values

$$
\mathbf{T}_{i=1}^{0} x_{i}=1, \mathbf{T}_{i=1}^{n} x_{i}=T\left(\mathbf{T}_{i=1}^{n-1} x_{i}, x_{n}\right)
$$

We can extend $T$ to a countable infinitary operation taking for any sequence $\left(x_{n}\right)_{n \in \mathbb{N}}$ from $[0,1]$ the values

$$
\mathbf{T}_{i=1}^{\infty} x_{i}=\lim _{n \rightarrow \infty} \mathbf{T}_{i=1}^{n} x_{i}
$$

Limit of the right side exists since the sequence $\left(\mathbf{T}_{i=1}^{n} x_{i}\right)_{n \in \mathbb{N}}$ is nonincreasing and bounded from below.

In [4], the following lemma is given.

Lemma 1. A Menger space $(S, \mathcal{F}, T)$ such that $\sup _{x<1} T(x, x)=1$ is a quasiuniformizable space $\left(S,\left(d_{\lambda}\right)_{\lambda \in J}, g\right)$, where $J=(0,1), g: J \rightarrow J$,

$$
d_{\lambda}(x, y)=\sup \left\{s \mid F_{x, y}(s) \leq 1-\lambda\right\}, \lambda \in J, x, y \in S .
$$

Every locally convex space $\left(X,\left(p_{i}\right)_{i \in I}\right)$, where $\left(p_{i}\right)_{i \in I}$ is the familly of seminorms on $X$, is a quasi-uniformizable space, where $g: I \rightarrow I$ is defined by $g(i)=i$, for every $i \in I$.

\section{Common fixed point theorem}

In this paper we prove a common fixed point theorem in some special class of quasi-uniformizable spaces. This theorem is a generalization of a theorem proved in [4]. 
Lemma 2. Let $(S, \mathcal{F}, T)$ be a Menger space and $T$ be a t-norm of H-type. Then $\left(S,\left(d_{\lambda}\right)_{\lambda \in J}, g\right), J=(0,1)$ is a quasi-uniformizable space such that for every $\alpha \in(0,1)$ there exists $\beta \in(0,1)$ such that for all $x_{1}, \ldots x_{n} \in S$

$$
d_{\alpha}\left(x_{1}, x_{n}\right) \leq d_{\beta}\left(x_{1}, x_{2}\right)+d_{\beta}\left(x_{2}, x_{3}\right)+\cdots+d_{\beta}\left(x_{n-1}, x_{n}\right) .
$$

Proof. Let $\alpha \in(0,1)$. Since $T$ is a t-norm of $H$-type for a given $\alpha \in(0,1)$, there exists $\beta \in(0,1)$ such that $\mathbf{T}_{i=1}^{n-1}(1-\beta)>1-\alpha$, for all $n \in \mathbb{N}$.

In order to prove (Z) we shall suppose that $\alpha_{1}, \alpha_{2}, \ldots \alpha_{n-1}, n \in \mathbb{N}$ is such that

$$
\begin{aligned}
d_{\beta}\left(x_{1}, x_{2}\right) & <\alpha_{1} \\
d_{\beta}\left(x_{2}, x_{3}\right) & <\alpha_{2} \\
& \ldots \\
d_{\beta}\left(x_{n-1}, x_{n}\right) & <\alpha_{n-1} .
\end{aligned}
$$

We need to prove $d_{\alpha}\left(x_{1}, x_{n}\right)<\alpha_{1}+\alpha_{2}+\cdots+\alpha_{n-1}$.

From (3) it follows

$$
\begin{aligned}
F_{x_{1}, x_{2}}\left(\alpha_{1}\right) & >1-\beta \\
F_{x_{2}, x_{3}}\left(\alpha_{2}\right) & >1-\beta \\
& \cdots \\
F_{x_{n-1}, x_{n}}\left(\alpha_{n-1}\right) & >1-\beta
\end{aligned}
$$

and

$$
\begin{aligned}
F_{x_{1}, x_{n}}\left(\alpha_{1}+\alpha_{2}+\cdots+\alpha_{n-1}\right) & \geq T\left(F_{x_{1}, x_{2}}\left(\alpha_{1}\right), F_{x_{2}, x_{n}}\left(\alpha_{2}+\cdots+\alpha_{n-1}\right)\right) \\
& \geq \underbrace{T(T(\ldots T}_{(n-2)-\text { time }}\left(F_{x_{1}, x_{2}}\left(\alpha_{1}\right) \ldots\left(F_{x_{n-1}, x_{n}}\left(\alpha_{n-1}\right)\right)\right) \\
& \geq \underbrace{T(T(\ldots T}_{(n-2)-\text { time }}(1-\beta, 1-\beta) \ldots, 1-\beta) \\
& =\mathbf{T}_{i=1}^{n-1} 1-\beta \\
& >1-\alpha
\end{aligned}
$$

so $d_{\alpha}\left(x_{1}, x_{n}\right)<\alpha_{1}+\alpha_{2}+\cdots+\alpha_{n-1}$ i.e. (2) holds.

Definition 2. Let $\left(S,\left(d_{\lambda}\right)_{\lambda \in I}, f\right)$ be a sequentially complete Hausdorff quasiuniformizable space. We say that the space satisfies condition $\left(^{*}\right)$, if for a given $\alpha \in I$ there exists $\beta \in I$ such that for all $x_{1}, \ldots, x_{n} \in S$

$$
d_{\alpha}\left(x_{1}, x_{n}\right) \leq d_{\beta}\left(x_{1}, x_{2}\right)+d_{\beta}\left(x_{2}, x_{3}\right)+\cdots+d_{\beta}\left(x_{n-1}, x_{n}\right) .
$$


Common fixed point theorem in quasi-uniformizable spaces

We observe that, a Menger space $(S, \mathcal{F}, T)$, where $T$ is a t-norm of H-type, satisfies the condition $(*)$.

Theorem 1. Let $\left(S,\left(d_{\lambda}\right)_{\lambda \in I}, f\right)$ be a sequentially complete Hausdorff quasiuniformizable space, family $\left(d_{\lambda}\right)_{\lambda \in I}$ satisfies the condition (2), $f: I \rightarrow I$, $L_{1}, L_{2}: S \rightarrow S$ continuous mapping, $L: S \rightarrow L_{1} S \cap L_{2} S$ a continuous mapping which commutes with $L_{1}$ and $L_{2}$, and the following conditions are satisfied:

(i) for every $i \in I$ there exists $q_{i}: \mathbb{R}^{+} \rightarrow[0,1)$ which is a non-decreasing function, for which $\varlimsup_{n \rightarrow \infty} q_{f^{n}(i)}(t)<1$, for every $t \in \mathbb{R}^{+}$and every $i \in I$ and

$$
d_{i}(L x, L y) \leq q_{i}\left(d_{f(i)}\left(L_{1} x, L_{2} y\right)\right) \cdot d_{f(i)}\left(L_{1} x, L_{2} y\right) \text { for every } x, y \in S
$$

(ii) there exists $x_{0} \in S$ such that for every $i \in I$

$$
\sup _{j \in O(i, f)} d_{j}\left(L x_{0}, L x_{1}\right) \leq K_{i} \quad\left(K_{i} \in \mathbb{R}^{+}\right)
$$

where $O(i, f)=\left\{f^{n}(i): n \in \mathbb{N}\right\}$, and the sequence $\left(x_{p}\right)_{p \in \mathbb{N}}$ is defined by

$$
L_{1} x_{2 n-1}=L x_{2 n-2} \text { and } L_{2} x_{2 n}=L x_{2 n-1},(n \in \mathbb{N}) \text {. }
$$

Then there exists $z \in S$ such that $L z=L_{1} z=L_{2} z$.

If for every $i \in I$

$$
\sup _{j \in O(i, f)} d_{j}\left(L^{3} x_{1}, L^{2} x_{0}\right) \leq M_{i},\left(M_{i} \in \mathbb{R}^{+}\right)
$$

then $L z$ is a common fixed point for $L, L_{1}$ and $L_{2}$, and $L z$ is the unique common fixed point for $L, L_{1}, L_{2}$ in the set

$$
\left\{u \mid u \in S,(\forall i \in I),\left(\exists V_{i} \in \mathbb{R}^{+}\right)\left(\sup _{j \in O(i, f)} d_{j}(L z, u) \leq V_{i}\right)\right\}
$$

Proof. Let $\left(x_{n}\right)_{n \in \mathbb{N}}$ be such sequence from $S$ that

$$
L_{2} x_{2 k}=L x_{2 k-1} \text { and } L_{1} x_{2 k-1}=L x_{2 k-2} \text {, for every } k \in \mathbb{N} \text {. }
$$

We will prove that $\left(L x_{n}\right)_{n \in \mathbf{N}}$ is a Cauchy sequence which means that for every $i \in I$ and every $\varepsilon>0$ there exists $n(\varepsilon, i) \in \mathbb{N}$ such that

$$
d_{i}\left(L x_{n}, L x_{n+p}\right)<\varepsilon, \text { for every } n \geq n(\varepsilon, i), \text { and every } p \in \mathbb{N}
$$

In order to prove (5), we will estimate

$d_{i}\left(L x_{2 k}, L x_{2 k-1}\right)$ and $d_{i}\left(L x_{2 k+1}, L x_{2 k}\right)$, for every $i \in I$ and every $k \in \mathbb{N}$. 
From condition (i) it follows that

$$
\begin{aligned}
& d_{i}\left(L x_{2 k}, L x_{2 k-1}\right) \\
& \quad \leq \quad q_{i}\left(d_{f(i)}\left(L_{2} x_{2 k}, L_{1} x_{2 k-1}\right)\right) \cdot d_{f(i)}\left(L_{2} x_{2 k}, L_{1} x_{2 k-1}\right) \\
& \quad=q_{i}\left(d_{f(i)}\left(L x_{2 k-1}, L x_{2 k-2}\right)\right) \cdot d_{f(i)}\left(L x_{2 k-1}, L x_{2 k-2}\right) \\
& \leq \quad q_{i}\left(d_{f(i)}\left(L x_{2 k-1}, L x_{2 k-2}\right)\right) \cdot q_{f(i)}\left(d_{f^{2}(i)}\left(L_{1} x_{2 k-1}, L_{2} x_{2 k-2}\right)\right) \\
& \quad \cdot \quad\left(d_{f^{2}(i)}\right)\left(L_{1} x_{2 k-1}, L_{2} x_{2 k-2}\right) \\
& \quad \cdots \\
& \quad q_{i}\left(d_{f(i)}\left(L x_{2 k-1}, L x_{2 k-2}\right)\right) \\
& \quad \cdot \prod_{s=0}^{2 k-3} q_{f^{s+1}(i)}\left(d_{f^{s+2}(i)}\left(L x_{2 k-s-1}, L x_{2 k-s-2}\right)\right) \cdot d_{f^{2 k-1}(i)}\left(L x_{1}, L x_{0}\right)
\end{aligned}
$$

and similarly

$$
\begin{aligned}
d_{i}\left(L x_{2 k+1}, L x_{2 k}\right) \leq & q_{i}\left(d_{f(i)}\left(L x_{2 k}, L x_{2 k-1}\right)\right) \\
& \cdot \prod_{s=0}^{2 k-2} q_{f^{s+1}(i)}\left(d_{f^{s+2}(i)}\left(L x_{2 k-s-1}, L x_{2 k-s-2}\right)\right) \\
& \cdot d_{f^{2 k}(i)}\left(L x_{1}, L x_{0}\right)
\end{aligned}
$$

Since $q_{i}(t)<1$ for every $i \in I$ and every $t \in \mathbb{R}^{+}$, it follows that for every $i \in I$ and every $n \in \mathbb{N}$

$$
d_{j}\left(L x_{n}, L x_{n-1}\right) \leq K_{i},
$$

for every $j \in O(i, f)$ and therefore

$$
\begin{aligned}
& d_{i}\left(L x_{2 k}, L x_{2 k-1}\right) \leq \prod_{s=0}^{2 k-2} q_{f^{s}(i)}\left(K_{i}\right) K_{i} \\
& d_{i}\left(L x_{2 k+1}, L x_{2 k}\right) \leq \prod_{s=0}^{2 k-1} q_{f^{s}(i)}\left(K_{i}\right) K_{i}
\end{aligned}
$$

where $f^{0}(i)=i$ for every $i \in I$.

Since $\lim _{n \rightarrow \infty} q_{f^{n}(i)}\left(K_{i}\right) \leq Q_{i}<1$, for all $i \in I$ there exists $n_{i} \in \mathbb{N}$ such that for some $S_{i} \in \mathbb{R}^{+}(i \in I)$ it follows

$$
d_{i}\left(L x_{n}, L x_{n-1}\right) \leq S_{i} Q_{i}^{n},
$$

for every $i \in I$.

From Lemma $\mathbf{2}$, for every $i \in I$ there exists $j \in I$ such that

$$
\begin{aligned}
d_{i}\left(L x_{n}, L x_{n+p}\right) \leq & d_{j}\left(L x_{n}, L x_{n+1}\right)+d_{j}\left(L x_{n+1}, L x_{n+2}\right)+\ldots \\
& \cdots+d_{j}\left(L x_{n+p-1}, L x_{n+p}\right) \\
\leq & S_{j} \sum_{i=n+1}^{\infty} Q_{j}^{i}
\end{aligned}
$$


From the condition $Q_{j}<1,(j \in I)$, it follows that the sequence $\left(L x_{n}\right)_{n \in \mathbf{N}}$ is a Cauchy sequence. Let

$$
z=\lim _{n \rightarrow \infty} L x_{n}=\lim _{n \rightarrow \infty} L x_{2 n-1}=\lim _{n \rightarrow \infty} L x_{2 n} .
$$

Since $L$ and $L_{1}$ are continuous functions, we have

$$
L_{1} z=\lim _{n \rightarrow \infty} L_{1} L x_{2 n+1}=\lim _{n \rightarrow \infty} L L_{1} x_{2 n+1}=L z
$$

and similarly $L_{2} z=L z$.

We are going to prove that $L z$ is a common fixed point for the mappings $L$, $L_{1}$ and $L_{2}$ if

$$
\sup _{j \in O(i, f)} d_{j}\left(L^{3} x_{1}, L^{2} x_{0}\right) \leq M_{i}
$$

for all $i \in I$.

First we shall prove that for every $i \in I$

$$
\sup _{j \in O(i, f)} d_{j}\left(L^{2} z, L z\right) \leq M_{i} .
$$

Since

$$
d_{j}\left(L^{2} z, L z\right)=\lim _{n \rightarrow \infty} d_{j}\left(L^{2} L x_{2 n+1}, L L x_{2 n}\right)
$$

it is enough to prove that for every $i \in I$

$$
d_{j}\left(L^{3} x_{2 n+1}, L^{2} x_{2 n}\right) \leq M_{i} \text { for every } n \in \mathbb{N} \text { and } j \in O(i, f) .
$$

The pairs $L, L_{1}$ and $L, L_{2}$ commute, and we obtain that

$$
\begin{aligned}
d_{j} & \left(L^{3} x_{2 n+1}, L^{2} x_{2 n}\right) \\
\leq & q_{j}\left(d_{f(j)}\left(L_{2}\left(L x_{2 n}\right), L_{1}\left(L^{2} x_{2 n+1}\right)\right)\right) d_{f(j)}\left(L_{2}\left(L x_{2 n}\right), L_{1}\left(L^{2} x_{2 n+1}\right)\right) \\
= & q_{j}\left(d_{f(j)}\left(L\left(L_{2} x_{2 n}\right), L^{2}\left(L_{1} x_{2 n+1}\right)\right)\right) d_{f(j)}\left(L\left(L_{2} x_{2 n}\right), L^{2}\left(L_{1} x_{2 n+1}\right)\right) \\
= & q_{j}\left(d_{f(j)}\left(L L x_{2 n-1}, L^{2} L x_{2 n}\right)\right) d_{f(j)}\left(L L x_{2 n-1}, L^{2} L x_{2 n}\right) \\
= & q_{j}\left(d_{f(j)}\left(L^{2} x_{2 n-1}, L^{3} x_{2 n}\right)\right) d_{f(j)}\left(L^{2} x_{2 n-1}, L^{3} x_{2 n}\right) \\
\leq & d_{f(j)}\left(L^{2} x_{2 n-1}, L^{3} x_{2 n}\right) \\
& \vdots \\
\leq & d_{f(j)}\left(L^{3} x_{1}, L^{2} x_{0}\right) \\
\leq & M_{i}
\end{aligned}
$$

for every $n \in \mathbb{N}$ since $f^{2 n}(j) \in O(i, f)$.

Now we have

$$
\begin{aligned}
d_{i}\left(L^{2} z, L z\right) \leq & q_{i}\left(d_{f(i)}\left(L_{1} L z, L_{2} z\right)\right) \cdot d_{f(i)}\left(L_{1} L z, L_{2} z\right) \\
= & q_{i}\left(d_{f(i)}\left(L^{2} z, L z\right)\right) \cdot d_{f(i)}\left(L^{2} z, L z\right) \\
& \vdots \\
\leq & q_{i}\left(M_{i}\right) q_{f(i)}\left(M_{i}\right) \ldots q_{f^{n}(i)}\left(M_{i}\right) M_{i}
\end{aligned}
$$


for every $i \in I$.

Since $\varlimsup_{n \rightarrow \infty} q_{f^{n}(i)}\left(M_{i}\right)<1$ it follows that $d_{i}\left(L^{2} z, L z\right)=0$ for every $i \in I$, which implies that $L z=L^{2} z$.

Hence, from $L z=L_{1} z=L_{2} z$ it follows that

$$
L^{2} z=L L_{1} z=L_{1} L z=L_{2} L z=L z
$$

which means that $L z$ is a common fixed point for the mappings $L, L_{1}$ and $L_{2}$.

Suppose that $y=L y=L_{1} y=L_{2} y$ and for every $i \in I$

$$
\sup _{j \in O(i, f)} d_{j}(L z, y) \leq V_{i} \quad\left(V_{i} \in \mathbb{R}^{+}\right) .
$$

We prove that $y=L z$. For every $i \in I$ we have

$$
\begin{aligned}
d_{i}(L z, y)= & d_{i}(L(L z), L y) \\
\leq & q_{i}\left(d_{f(i)}\left(L_{1}(L z), L_{2} y\right)\right) \cdot d_{f(i)}\left(L_{1}(L z), L_{2} y\right) \\
= & q_{i}\left(d_{f(i)}(L z, y)\right) \cdot d_{f(i)}(L z, y) \\
& \vdots \\
\leq & q_{i}\left(d_{f(i)}(L z, y)\right) \ldots q_{f^{n}(i)}\left(d_{f^{n+1}(i)}(L z, y)\right) \cdot d_{f^{n+1}(i)}(L z, y) \\
\leq & q_{i}\left(V_{i}\right) q_{f(i)}\left(V_{i}\right) \ldots q_{f^{n}(i)}\left(V_{i}\right) V_{i}
\end{aligned}
$$

Since $\varlimsup_{n \rightarrow \infty} q_{f^{n}(i)}\left(V_{i}\right)<1$ it follows that $d_{i}(L z, y)=0$ for every $i \in I$, which means that $y=L z$.

Corollary 1. Let $(S, \mathcal{F}, T)$ be a complete Menger space and t-norm $T$ is of $H$-type, $f:(0,1) \rightarrow(0,1), L_{1}, L_{2}: S \rightarrow S$ continuous mappings, $L: S \rightarrow$ $L_{1} S \cap L_{2} S$ a continuous mapping which commutes with $L_{1}$ and $L_{2}$, and the following conditions are satisfied:

(a) for every $\lambda \in(0,1)$, there exists a right continuous, non-decreasing mapping

$$
q_{\lambda}: \mathbb{R}^{+} \rightarrow[0,1) \text { for which } \varlimsup_{n \rightarrow \infty} q_{f^{n}(\lambda)}(t)<1 \text { for every } t \in \mathbb{R}^{+}
$$

and for every $\lambda \in(0,1)$, every $r>0$ and every $x, y \in S$

$$
F_{L_{1} x, L_{2} y}(r)>1-f(\lambda) \Rightarrow F_{L x, L y}\left(q_{\lambda}(r) r\right)>1-\lambda .
$$

(b) there exists $x_{0} \in S$ such that for every $\lambda \in(0,1)$, there exists $K_{\lambda} \in \mathbb{R}^{+}$such that for every $n \in \mathbb{N}$

$$
F_{L x_{0}, L x_{1}}\left(K_{\lambda}\right)>1-f^{n}(\lambda)
$$

where the sequence $\left(x_{n}\right)_{n \in \mathbb{N}}$ is defined by

$$
L_{1} x_{2 n-1}=L x_{2 n-2} \text { and } L_{2} x_{2 n}=L x_{2 n-1} \quad(n \in \mathbb{N}) .
$$

Then, there exists $z \in S$ such that

$$
L z=L_{1} z=L_{2} z .
$$


If, in addition, for every $\lambda \in(0,1)$ there exists $M_{\lambda} \in \mathbb{R}^{+}$such that for every $n \in \mathbb{N}$

$$
F_{L^{3} x_{1}, L^{2} x_{0}}\left(M_{\lambda}\right)>1-f^{n}(\lambda)
$$

then, $L z$ is a common fixed point for the mapping $L, L_{1}$ and $L_{2}$, which is the unique common fixed point in the set

$$
\left\{u \mid u \in S,(\forall \lambda \in(0,1))\left(\exists P_{\lambda} \in \mathbb{R}^{+}\right)(\forall n \in \mathbb{N})\left(F_{L z, u}\left(P_{\lambda}\right)>1-f^{n}(\lambda)\right)\right\} .
$$

Proof. We have only to prove that condition (6) implies that for every $\lambda \in(0,1)$ and every $x, y \in S$

$$
d_{\lambda}(L x, L y) \leq q_{\lambda}\left(d_{f(\lambda)}\left(L_{1} x, L_{2} y\right)\right) \cdot d_{f(\lambda)}\left(L_{1} x, L_{2} y\right)
$$

Suppose that $d_{f(\lambda)}\left(L_{1} x, L_{2} y\right)<r$. Then, $F_{L_{1} x, L_{2} y}(r)>1-f(\lambda)$ and by $(6)$ we obtain that $F_{L x, L y}\left(q_{\lambda}(r) r\right)>1-\lambda$. Hence, $d_{\lambda}(L x, L y)<q_{\lambda}(r) r$ and since $q_{\lambda}$ is right-continuous, the inequality (7) holds.

From Lemma $\square$ it follows that for every $\alpha \in(0,1)$ there exists $\beta \in(0,1)$ such that for every $x_{1}, \ldots, x_{n} \in S$

$$
d_{\alpha}\left(x_{1}, x_{n}\right) \leq d_{\beta}\left(x_{1}, x_{2}\right)+d_{\beta}\left(x_{2}, x_{3}\right)+\cdots+d_{\beta}\left(x_{n-1}, x_{n}\right)
$$

and all conditions of the previous theorem are satisfied.

\section{Acknowledgment}

The work was supported by the Ministry of Education and Science of the Republic of Serbia under grants 174009.

\section{References}

[1] Hadžić O., A fixed point theorem in Menger spaces. Publ. Inst. Math. Beograd T 20 (1979), 107-112.

[2] Hadžić O., On a common fixed point in quasi-uniformizable spaces. Review of Research Faculty of Science-University of Novi Sad, Vol. 13 (1983), 31-39.

[3] Hadžić O., On Common Fixed Points in Uniformizable Spaces. Anal. Num. Appr., 12, 1(1983), 45-54.

[4] Hadžić O., Pap E., Fixed Point Theory in Probabilistic Metric Spaces. Theory in Probabilistic Metric Spaces. Dordrecht: Kluwer Academic Publishers, 2001.

[5] Klement E.P., Mesiar R., Pap E., Triangular Norms, Trends in Logic 8. Dordrecht: Kluwer Academic Publishers, 2000.

[6] Menger K., Statistical metric. Proc. Nat. Acad. U.S.A., 28 (1942), 535-537.

[7] Schweizer B., Sklar A., Probabilistic metric spaces. New York: Elsevier NorthHolland, 1983.

[8] Tan D.H., On the contraction principle in uniformizable spaces, Acta Mathematica Vietnamica, Tom 5 (1980), 88-99.

[9] Žikić T., Existence of fixed point in fuzzy structures, PhD Thesis 2002., University of N. Sad, Faculty of Sciences and Mathematics.

Received by the editors December 18, 2010 